\title{
Acumulación de capacidades tecnológicas locales en un grupo industrial siderúrgico en México
}

\author{
Javier Jasso V. ${ }^{*}$ \\ Rodrigo Ortega**
}

\section{Resumen}

Las empresas construyen capacidades tecnológicas a través de procesos de aprendizaje; de modo que el aprendizaje tecnológico se concibe como el proceso dinámico de adquisición de capacidades tecnológicas. Los procesos de construcción de capacidades tecnológicas dependen de un conjunto de factores vinculados con los flujos de conocimiento dentro y entre la empresa y su entorno; en particular, el contexto en el cual compiten afecta los procesos de acumulación. Asimismo, implican un proceso de desarrollo de capacidades internas que se construyen a partir de esfuerzos propios y de la experiencia y conocimientos disponibles.

El presente trabajo analiza el proceso de acumulación utilizando una matriz de capacidades tecnológicas que mide las funciones técnicas de inversión, producción y soporte en un grupo industrial (siderúrgico) mexicano ${ }^{1}$, en el periodo 1999-2003.

La principal conclusión es que en dicho grupo corporativo se han emprendido procesos de aprendizaje internos y locales que le han permitido construir capacidades tecnológicas de manera gradual y diferenciada (adquisición, asimilación y adaptación), y mejorar así sus niveles de productividad.

Palabras clave: Aprendizaje tecnológico, funciones técnicas, capacidades.

Fecha de recepción: 14-03-2006

Fecha de aceptación: 11-05-2007

* Profesor investigador de la Facultad de Contaduría y Administración, UNAM. Correo electrónico: jassov@correo.posgrado.unam.mx

** Catedrático del Departamento de Ciencias Económico Administrativo del Instituto Tecnológico de San Luis Potosí. Correo electrónico: rortegar@cablecomslp.com.mx

${ }^{1}$ Grupo San es el nombre ficticio que hemos dado a la empresa estudiada, con la finalidad de mantener la confidencialidad de la información proporcionada por la empresa, en que agradecemos su colaboración y apoyo. 
Javier Jasso V. y Rodrigo Ortega

\title{
Learning and Accumulating Technological Capacity in an Industrial Group from the Iron and Steel Sector
}

\begin{abstract}
Learning and accumulating technological ability help to shape the world of current knowledge, thus being fundamental for competitiveness between countries, regions and companies. Such learning and accumulation of ability implies constructing internal ability through the efforts and available experience and knowledge developed by a particular company or any other institution leading to innovation. It thereby represents one of the fundamental elements of competitiveness.
\end{abstract}

The regularity with which a company incorporates radical or incremental improvements to its products, processes or organizational schemes is a decisive factor in the growth of its productivity.

This work analyses the process of innovation from an internal perspective to clarify how an industrial group from the iron and steel sector develops its technological ability through exercising its technical investment, production and support functions.

Key Words: Technological ability, technological learning, technical investment functions, production and support.

\section{Introducción}

$\mathrm{D}$ esde hace ya más de tres décadas se ha examinado la importancia del aprendizaje en el desarrollo de la tecnología y de la industria (Rosenberg, 1979). Los economistas han tratado de explicar cómo el aprendizaje afecta a la productividad (Arrow, 1962) o a la estructura industrial (Dosi et al., 1988). El aprendizaje dentro de las empresas ha sido una característica de la teoría de la firma (Cyret y March, 1963) y juega un papel decisivo en la teoría de la administración estratégica de las capacidades dinámicas (Dosi et al., 2000). En el caso de las empresas en países en desarrollo, la literatura ha analizado estos temas centrando la atención en los procesos de aprendizaje; en particular, en lo relativo a la construcción y acumulación gradual de capacidades tecnológicas, tomando como punto de partida la tecnología adquirida en otros países.

El aprendizaje y la construcción de capacidades tecnológicas, a nivel de la empresa, significa un proceso dinámico de obtención y creación de capacidades internas y de los conocimientos disponibles en otras empresas e instituciones. Los procesos de 
construcción de capacidades tecnológicas dependen, por lo tanto, de un conjunto de factores relacionados con los flujos de conocimiento dentro de la empresa y entre la empresa y el contexto en el cual compite.

La idea de que el aprendizaje tecnológico se relaciona con la capacidad de la empresa para adquirir tecnología, para absorberla y adaptarla a las condiciones locales, se favorece en la medida en que las relaciones sociales en la empresa permiten sustentar un proceso colectivo de aprendizaje tecnológico: equipos humanos que pueden tomar sus propias decisiones, nuevas formas creativas de hacer el trabajo, una atmósfera flexible, un intercambio permanente de los conocimientos y experiencias adquiridos en la ejecución de los procesos, aprendizajes abiertos a sistemas externos, diálogo permanente como base para una mentalidad abocada a la solución de problemas. El aprendizaje y la capacidad innovadora no se relacionan sólo con el ambiente interno de una empresa, sino que dependen también del ambiente externo y de los cambios en la demanda. ${ }^{2}$

Desde la perspectiva de los enfoques del aprendizaje y las capacidades tecnológicas, esta investigación examina las actividades desarrolladas en cada una de las áreas productivas del Grupo San, a fin de estudiar la manera en que la tecnología se ha articulado con las formas de organizar los factores de la producción y las actividades, mecanismos y formas de aprendizaje tecnológico, que se ha experimentado para adquirir, integrar y dominar la tecnología actual.

Se emplea para tal propósito la metodología del estudio de caso ${ }^{3} \mathrm{y}$, como instrumentos para obtener la información, la observación directa en la planta productiva, las entrevistas no estructuradas y semi-estructuradas complementadas con referencias para su clasificación y correspondencia.

El argumento guía del trabajo es que, en el periodo analizado, se han emprendido procesos de aprendizaje interno que facilitan una adecuada capacidad de absorción

\footnotetext{
${ }^{2}$ El acceso que tiene cada empresa al desarrollo de una capacidad productiva tiene un efecto importante en los procesos de aprendizaje no sólo para el individuo, sino también para la organización. Sin embargo, para que el conocimiento que se deriva del manejo de nuevas tecnologías (maquinaria y equipo) se encuentre al alcance de los usuarios, es necesario que éstos cuenten con los manuales que la codifican, es decir, que se encuentre a su alcance ese conocimiento codificado (Malecki, 1997).

${ }^{3}$ El estudio de caso es uno de los varios caminos para hacer investigación social (Yin, 1994). Está diseñado para mostrar los detalles desde un punto de vista de los participantes a través de múltiples fuentes de datos (Tellis, 1997b). Los estudios de caso cumplen con los tres principios básicos de la metodología cualitativa: son descriptivos, entendibles y explicativos (Tellis, 1997a). Han sido aplicados en investigaciones de negocios, situaciones organizacionales, educación, desarrollo de tecnología y problemas sociales, entre otros (Yin, 1994).
} 
tecnológica (adquisición, asimilación, adaptación y cambio menor), con lo que se logran importantes niveles de productividad. Por otra parte, el artículo está organizado en cuatro apartados. En el primero se exponen los fundamentos teóricos; en el segundo se explica la metodología practicada; en el tercero se muestran los resultados del estudio y, finalmente, en el cuarto se hace una recapitulación y se presentan las conclusiones.

\section{De la asimilación automática a la acumulación de capacidades}

Hasta avanzados los años setenta existía la idea, bastante difundida, de que los países en desarrollo eran puramente receptores pasivos de la tecnología ${ }^{4}$ avanzada del mundo industrializado. Esta postura fue desafiada vigorosamente por una serie de estudios empíricos sobre los procesos de aprendizaje y la adquisición de ciertas capacidades tecnológicas al nivel de empresa. En dichos estudios se documentó una vasta evidencia empírica que mostraba que el conocimiento tecnológico no es fácil de imitar ni transferir entre empresas, porque tal conocimiento es tácito y acumulativo. ${ }^{5}$ Lo anterior condujo a la comprensión de que la transferencia de tecnología sólo se podría hacer efectiva cuando las empresas receptoras vinculaban los procesos de transferencia con procesos de aprendizaje y desarrollo de capacidades internas. La empresa no es una "caja negra” sino una caja abierta y compleja. ${ }^{6}$ Así, la empresa que aplica una tecnología importada por primera vez, aun cuando se trata de un cuerpo de conocimiento muy difundido en el mundo desarrollado, requiere aprender y desplegar un esfuerzo consciente (Lall, 1987). Más aun: ninguna tecnología puede ser aplicada en una forma completamente dada, ya que siempre son necesarios cambios para satisfacer las escalas, las materias primas, el clima, las habilidades y las necesidades de los mercados locales. ${ }^{7}$

Las empresas realizan en sus rutinas ${ }^{8}$ innovaciones "menores" en sus procesos, productos y sistemas. Al respecto, Kim (1997: 23) afirma que "la tecnología puede ser

\footnotetext{
${ }^{4}$ Definimos a la tecnología como el conjunto de conocimientos que son incorporados al sistema productivo para el control y mejoramiento de las actividades de gestión y organización del trabajo, de diseño, de producción y comercialización de un bien o servicio (Jasso, 2004).

${ }^{5}$ Entre otros están los trabajos pioneros de Katz, (véase entre otros el texto de 1989, en donde se citan sus trabajos anteriores de los años setenta).

${ }^{6}$ Para una revisión más detallada, y acerca de la caracterización de la empresa como una caja abierta y compleja, véase Jasso (2004).

${ }^{7}$ Para Lall (1996: 190) gran parte de la bibliografía tradicional, teórica y empírica, ha pasado por alto la necesidad de estudiar la actividad tecnológica y su producción en los países en desarrollo. En la teoría neoclásica se prescinde de ese problema, mientras que en la teoría del comercio internacional se considera que la tecnología es de libre acceso para todos los países y empresas.

${ }^{8}$ Las rutinas incluyen actividades de imitación, copia, selección, asimilación, transferencia de conocimientos, técnicas y procedimientos entre otros.
} 
transferida a una empresa desde el exterior, o mediante la difusión local, pero no así la habilidad para usarla efectivamente. Esta habilidad sólo se puede adquirir por el esfuerzo tecnológico interno”.

En los países en desarrollo dos grandes proyectos llevados a cabo entre finales de los 70 y comienzos de los años 80 marcan un parte-aguas acerca del estudio de aprendizaje y acumulación de capacidades tecnológicas. El primero, denominado "Programa de Investigación en Ciencia y Tecnología” y financiado por IDB/ECLA, realizó una investigación comparativa al nivel de empresa de la industria metalmecánica de seis países latinoamericanos. El segundo, "La adquisición de capacidades tecnológicas”, financiado por el Banco Mundial que incluyó a empresas de América Latina, India y Corea del Sur.

Estos estudios mostraron que muchas empresas de países en desarrollo han experimentado importantes procesos de aprendizaje tecnológico, es decir, que no eran simples receptores de la tecnología. También evidenciaron comportamientos diferenciados por tipo de empresa, naturaleza del proceso de producción y estructura de mercado. Posteriormente, Lall (1992), y más tarde Bell y Pavitt (1995), partiendo de la propuesta del primero presentaron una taxonomía de capacidades tecnológicas ${ }^{9}$ en las empresas, basada en las capacidades tecnológicas operacionalizadas por funciones técnicas.

Desde la perspectiva de los enfoques del aprendizaje y las capacidades tecnológicas, esta investigación examina las actividades desarrolladas en cada una de las áreas productivas de un grupo industrial del sector siderúrgico, a fin de estudiar la manera en que la tecnología se ha articulado con las formas de organizar los factores de la producción y las actividades, ${ }^{10}$ mecanismos ${ }^{11} \mathrm{y}$ formas de aprendizaje tecnológico, que se han experimentado para adquirir, integrar y dominar la tecnología actual.

\footnotetext{
${ }^{9}$ La taxonomía de Bell y Pavitt $(1993,1995)$ clasifica las principales capacidades tecnológicas a partir de cuatro funciones técnicas: dos básicas y dos de apoyo. Las primeras son: 1) actividades de inversión y 2) actividades de producción. Las dos funciones de apoyo son 1) el desarrollo de vínculos con empresas e instituciones y 2) la producción de bienes de capital. Los niveles de capacidades tecnológicas se definen por el grado de dificultad de las actividades. Éstas van desde los niveles más básicos de las capacidades de producción rutinaria, hasta tres niveles (básico, intermedio y avanzado) de profundidad de las capacidades de innovación.

${ }^{10}$ El concepto "actividades de aprendizaje" se refiere a aquellas actividades que desarrolla la empresa a través de las cuales puede aprender. Es un concepto que no supone resultados. Algunas de las actividades de aprendizaje son: Producción, ingeniería, monitoreo de experiencias internas a nivel de planta, investigación y desarrollo, adaptación, ingeniería inversa, análisis de productos de competidores, visita a establecimiento de los competidores, licenciamiento.

${ }^{11}$ Algunos autores usan el concepto "mecanismos de aprendizaje”, el cual supone que la empresa desarrolló una actividad y ésta funcionó, de tal forma que se convirtió en un mecanismo reconocido.
} 


\section{Estudios y métodos de análisis de las capacidades tecnológicas}

En esta sección se registran algunos de los estudios y métodos para analizar las capacidades tecnológicas de las empresas.

\subsection{El estudio de las capacidades tecnológicas}

La mayoría de esos estudios emplea las aportaciones de Bell y Pavitt (1992) y, en especial, la taxonomía de Lall (1992) quien propone formas de clasificar las capacidades tecnológicas desarrolladas por la empresa con el fin de asimilar, adaptar y mejorar la tecnología adquirida. La taxonomía señalada distingue capacidades de inversión, ${ }^{12}$ de producción ${ }^{13}$ y de soporte. ${ }^{14}$

Estas capacidades se miden de acuerdo al nivel de aprendizaje e innovación. En el nivel primario (básicas), la empresa adquiere habilidades básicas; en el nivel medio (intermedias), alcanza habilidades secundarias (es decir, de imitación), y en el nivel más elevado (avanzadas), logra las habilidades innovativas más altas.

Algunas investigaciones que buscan identificar las fuentes de aprendizaje y las actividades tecnológicas de las empresas se basan en encuestas realizadas especialmente con este objeto, mientras que otras lo hacen en estadísticas oficiales. En el primer grupo destacan los trabajos siguientes: Westphal, Kritayakirana y otros (1990), realizado con una muestra de cien empresas tailandesas; Romijn (1999), centrado en la industria pakistaní de bienes de capital; Wignaraja (2001), con una muestra de empresas textiles y del vestido en las islas Mauricio, y Tremblay (1998), basado en una muestra de la industria papelera canadiense. La complejidad de los aspectos analizados en la construcción del índice es distinta en cada estudio.

Romijn (1999), por ejemplo, mide las capacidades tecnológicas en función del grado de complejidad involucrado en la fabricación de los bienes pertinentes, basando su enfoque en los mecanismos de aprendizaje de la industria metalmecánica a pequeña

${ }^{12}$ Las capacidades de inversión son las habilidades necesarias para identificar, preparar y obtener tecnología para el diseño, la construcción, el equipamiento y el personal de un nuevo proyecto (Lall, 1992).

${ }^{13}$ Las capacidades de producción van desde las habilidades básicas (control de calidad, operación, mantenimiento) hasta las más avanzadas (adaptación, mejora) y las más exigentes (investigación, diseño, innovación). Estas habilidades permiten no sólo operar y mejorar las tecnologías, sino también efectuar esfuerzos internos para absorber o imitar la tecnología comprada a otras empresas (Lall, 1992).

${ }^{14}$ Las capacidades de vinculación (o soporte) son las habilidades necesarias para el intercambio de información, tecnología y destrezas entre empresas (proveedores, subcontratistas, consultores, instituciones tecnológicas); influyen tanto en la eficiencia productiva de la empresa como en la difusión de la tecnología en la industria (Lall, 1992). 
escala. Asimismo, señala que la “diversificación hacia arriba” en esta industria ocurre mediante un proceso de aprendizaje por imitación de diseños y reproducción de bienes de fabricación cada vez más compleja; de forma tal que en un mismo parque de maquinaria y equipos es posible fabricar productos distintos, con diferentes grados de complejidad, dependiendo del nivel técnico de las operaciones realizadas. Por lo tanto, la manufactura de los distintos productos elaborados con un mismo parque de maquinaria puede requerir diferentes grados de habilidades. Por otra parte, en el trabajo citado se desarrolla un indicador de capacidad de producción, más que de innovación o de inversión; se reúne una muestra de 50 productos de la industria local; se diseña un sistema para identificar el nivel de capacidad de producción incorporado en cada bien, y se otorga una calificación al grado de complejidad tecnológica (habilidades y conocimiento) de cada una de las operaciones necesarias para fabricar el producto.

El índice tecnológico de Wignaraja (1998 y 2001) retoma la taxonomía de Lall, pero se estima a partir de dos capacidades tecnológicas, las de producción y las de vinculación, debido a la falta de datos para las de inversión. Examina 40 empresas de la industria del vestido por medio de una encuesta. La categoría de capacidades de producción está representada en el sistema de puntajes por diez actividades técnicas que van desde las tareas comunes de la ingeniería de procesos (tasas de rechazo, estatus según las normas de calidad de los productos industriales —ISO 9000—) a las de ingeniería de producto (copiado, mejora o introducción de nuevos productos). El incremento de la productividad también se incluyó en esta categoría. La categoría de capacidades de vinculación está representada por dos actividades técnicas: la transferencia de tecnología a través de subcontratistas y aquélla por contacto con empresas clientes del extranjero. ${ }^{15}$

Dutrenit y Capdevielle (1993) examinan la evolución de la manufactura, utilizando la clasificación de trayectorias tecnológicas de Pavitt (1984), que incluye una evaluación de las capacidades tecnológicas de las empresas. Para ello utilizan tres variables: las remuneraciones medias (como aproximación a la tecnología de habilidades), la inversión en maquinaria y equipo (como aproximación a la tecnología dura) y la investigación y desarrollo (como aproximación a la tecnología blanda).

Los trabajos aludidos enfrentaron, como bien se especifica en ellos, enormes dificultades de información para construir sus índices de capacidades, y se llevaron a cabo en gran medida mediante aproximaciones.

${ }^{15}$ Cada una de las 12 actividades técnicas que se han mencionado puede ser calificada en distintos niveles, que a su vez reflejan niveles de competencia diferentes en esa capacidad. Así, la posición que ocupe la empresa dependerá del total de puntos que logre de un puntaje máximo de 24, resultado que se normaliza entre 0 y 1. 


\subsection{Estudios de caso y teóricos de investigación}

La medición de las capacidades construidas parte del estudio de caso y se utilizan técnicas de recolección de la observación, ${ }^{16}$ la entrevista no estructurada, la entrevista semiestructurada, y el análisis de documentos.

El proceso metodológico inició con la observación directa y entrevistas no estructuradas (pláticas informales), ${ }^{17}$ con el gerente de recursos humanos y los superintendentes de acería y laminación. Se realizaron diversas visitas a la planta, apoyados en informantes clave de cada área. También se recopiló información documental, así como registros de calidad, capacitación, seguridad industrial y datos generales de la empresa. ${ }^{18}$ Esta información inicial, permitió diseñar una logística para la recolección de datos, ya que conociendo los procesos, identificadas las áreas productivas y las personas a entrevistar, se estableció un programa a seguir durante las entrevistas semi-estructuradas posteriores. ${ }^{19}$ En esta parte del trabajo de campo hubo necesidad

${ }^{16}$ La técnica de la observación proporciona información adicional sobre el objeto de estudio, al permitir obtener datos sobre aspectos que son más fáciles de percibir visualmente que a través de la comunicación oral. Para Yin (1994) las observaciones pueden corresponder a actividades de recolección de datos formales o casuales. Son formales cuando se desarrollan con base a un protocolo establecido donde el investigador mide la incidencia de cierto tipo de conductas en ciertos periodos durante el trabajo de campo. Con menos formalidad las observaciones directas pueden conducirse a lo largo de las visitas al objeto de estudio, incluyendo aquellas ocasiones en las que se obtuvo otro tipo de evidencia, como por ejemplo el desarrollo de las entrevistas. El realizar una visita al lugar del caso de estudio en cuestión da oportunidad a la observación directa (Yin, 1994) y en este caso fueron repetidas. Para efectos de esta investigación se realizó la observación directa casual propuesta por Yin (1994), la cual se llevó a cabo sin formalidades y evitando que las personas se sintieran bajo estudio, con lo que podrían cambiar su conducta habitual.

${ }^{17} \mathrm{El}$ acercamiento inicial con la empresa se hizo mediante una entrevista no estructurada con el director general. En ella se le explicaron los objetivos del estudio y se le pidió consentimiento para tener acceso a las instalaciones y facilidades para recolectar la información.

Las entrevistas no estructuradas (pláticas informales) se aplicaron en las etapas iniciales en las áreas a investigar y para corroborar la información; en ellas los aspectos que se analizaron procedieron principalmente de los entrevistados. Esta técnica fortaleció a la observación, ya que a través de las pláticas con las personas se corroboraron muchas de las observaciones.

${ }^{18}$ Para el estudio de caso, el uso más importante de los documentos es corroborar y aumentar la evidencia de otras fuentes. Los documentos son útiles para verificar la escritura correcta de nombres o títulos de instituciones que sean mencionados en la entrevista, así como para inferir cuestiones no evaluadas con anterioridad (Yin, 1994).

${ }^{19}$ Una de las fuentes de información más importante del estudio de caso es la entrevista semiestructurada. Mediante ésta individuos claves para la investigación pueden aportar datos y opiniones sobre el fenómeno de estudio. El entrevistado es un informante y, además de proveer aspectos relevantes, sugiere fuentes adicionales que puedan corroborar la evidencia (Yin, 1994). Se utilizaron las entrevistas semiestructuradas por la flexibilidad que tiene el entrevistador de usar una guía de preguntas, y poder en un momento dado, introducir preguntas adicionales para precisar u obtener mayor información sobre el tema tratado. 
de analizar en detalle las actividades desplegadas para poder hacer la distinción entre innovativas básicas, intermedias o avanzadas. Lo anterior se logró verificando conforme a la descripción y experiencia de los informantes, la aportación al cambio que resultó de cada una de las actividades ejecutadas en los procesos. ${ }^{20}$

Para analizar los procesos de aprendizaje se seleccionaron las actividades en las cuales la empresa realizó importantes esfuerzos por aprender y, a la vez, produjeron cambios técnicos en alguna de las funciones técnicas de inversión, producción o soporte. Asimismo, se determinó el nivel de innovación alcanzado de acuerdo con la taxonomía utilizada y las formas de aprendizaje practicadas en el desarrollo de dichas actividades.

El criterio utilizado para determinar el nivel de capacidades tecnológicas fue identificar las habilidades que se han desarrollado en las áreas productivas, a través de, las actividades que se hacen para mejorar los procesos y productos, para adaptar la tecnología y para generar y administrar el cambio técnico con el fin de tener competitividad en el mercado.

La conceptualización de las funciones de inversión, producción y soporte, que se utilizó para una identificación más precisa, clasificación y determinación del nivel de innovación de las actividades y resultados reportados por cada área productiva, se presentan en las tabla 1 y 2.

Tabla 1

Variables y definiciones de las funciones técnicas

\begin{tabular}{|l|l|}
\hline \multicolumn{1}{|c|}{ Variables } & \multicolumn{1}{c|}{ Definición } \\
\hline Toma de decisiones y control & $\begin{array}{l}\text { Son aquéllas que se requieren antes de crear nuevas instalaciones o expandir la planta } \\
\text { existente. Incluye, las capacidades para identificar necesidades potenciales, preparar y obtener la } \\
\text { tecnología necesaria y habilidades para diseñar, construir, equipar y conseguir personal calificado. } \\
\text { Habilidades para determinar el costo de la inversión del proyecto, su conveniencia, el tamaño de } \\
\text { planta, la diversidad de productos, las características de la tecnología, la búsqueda de fuentes } \\
\text { de tecnología, la negociación de contratos y la logística de abastecimientos. }\end{array}$ \\
\hline $\begin{array}{l}\text { proyecto } \\
\text { organización de la producción }\end{array}$ & $\begin{array}{l}\text { Se definen como las habilidades para lograr la competitividad sostenida que requiere el } \\
\text { cambio técnico después de la inversión inicial en las instalaciones de producción. Se refieren } \\
\text { a la generación y administración de cambio técnico en los procesos y la organización de la } \\
\text { producción, así como en los productos. }\end{array}$ \\
\hline Centradas en el producto & $\begin{array}{l}\text { Son las habilidades que contribuyen al proceso de cambio técnico, esto es, son habilidades que } \\
\text { permiten a las empresas recibir y transmitir información, conocimientos, experiencia y tecnología } \\
\text { de agentes localizados en el medio externo (proveedores, clientes, socios, competidores, } \\
\text { ferias tecnológicas, revistas especializadas, patentes, subcontratistas, empresas consultoras } \\
\text { tecnológicas, escuelas técnicas, instituciones universitarias públicas y privadas). }\end{array}$ \\
\hline Dinculación externa e internarrollo de equipo &
\end{tabular}

Fuente: Elaboración propia.

${ }^{20}$ Con el fin de corroborar la información obtenida en las entrevistas se formularon las mismas preguntas y se aplicaron las mismas guías con los diversos informantes, lo que permite garantizar la validez y confiabilidad de la investigación, así como tener diferentes puntos de vista del mismo hecho. 
Al utilizar estos conceptos en la determinación del tipo de función hubo necesidad de hacer analogías y utilizar ejemplos, para los casos en que la terminología no coincidía totalmente con las actividades reportadas. Sin embargo, a medida que se fue avanzando en las entrevistas, hubo mayor facilidad para distinguir las funciones de inversión. La tarea de hacer el recuento y distinción de las actividades realizadas, para lograr una mejor aproximación, requirió de analizar en detalle los reportes internos, a fin de obtener la mayor evidencia posible de este tipo de funciones (véanse tablas 1 y 2).

Tabla 2

Niveles de las funciones técnicas

\begin{tabular}{|c|c|c|}
\hline Nivel básico & Nivel intermedio & Nivel avanzado \\
\hline $\begin{array}{l}\text { Estudios de factibilidad } \\
\text { Selección de tecnología } \\
\text { Selección de proveedores } \\
\text { Programación de actividades }\end{array}$ & $\begin{array}{l}\text { Búsqueda, evaluación y selección de tecnología } \\
\text { Búsqueda, evaluación y selección de proveedores } \\
\text { Negociación con proveedores } \\
\text { Administración del proyecto }\end{array}$ & $\begin{array}{l}\text { Desarrollo de nuevos } \\
\text { sistemas de producción y } \\
\text { componentes }\end{array}$ \\
\hline $\begin{array}{l}\text { Estudios de factibilidad } \\
\text { Búsqueda de equipo } \\
\text { Ingeniería básica }\end{array}$ & $\begin{array}{l}\text { Ingeniería de detalle } \\
\text { Adquisición de equipo } \\
\text { Estudios del medio ambiente } \\
\text { Administración y seguimiento del proyecto } \\
\text { Designación del grupo de trabajo } \\
\text { Reclutamiento y Capacitación } \\
\text { Puesta en marcha }\end{array}$ & $\begin{array}{l}\text { Diseño de procesos y } \\
\text { desarrollo de investigación } \\
\text { relacionada }\end{array}$ \\
\hline $\begin{array}{l}\text { Adaptaciones menores al proceso } \\
\text { Implementación de mejoras incrementales } \\
\text { Formación de grupos trabajo } \\
\text { Mejora del layout } \\
\text { Programación de la producción } \\
\text { Mantenimiento productivo total } \\
\text { Mejorar el aprovechamiento de la materia prima } \\
\text { Mejorar el rendimiento de materiales indirectos }\end{array}$ & $\begin{array}{l}\text { Rediseño y/o diseño de partes del proceso, } \\
\text { ensamble y/o manufactura } \\
\text { Validación de procesos } \\
\text { Estiramiento de la capacidad de producción } \\
\text { Manufactura esbelta } \\
\text { Sistemas de calidad y mejora continua }\end{array}$ & Innovaciones en procesos \\
\hline $\begin{array}{l}\text { Obtener la certificación de la planta } \\
\text { Adaptaciones menores al producto de acuerdo } \\
\text { a necesidades del cliente } \\
\text { Mejorar las especificaciones del producto } \\
\text { Mejoras incrementales en la calidad del } \\
\text { producto }\end{array}$ & Diseño incremental de los productos & $\begin{array}{l}\text { Diseño de características } \\
\text { básicas de nuevos } \\
\text { productos } \\
\text { Innovaciones de productos }\end{array}$ \\
\hline $\begin{array}{l}\text { Relación con clientes a través de las } \\
\text { especificaciones del producto } \\
\text { Búsqueda y negociación con proveedores de } \\
\text { material indirecto } \\
\text { Búsqueda de vínculos con instituciones locales } \\
\text { para entrenamiento de personal }\end{array}$ & $\begin{array}{l}\text { Transferencia de tecnología a proveedores } \\
\text { locales para incrementar eficiencia, calidad y } \\
\text { abastecimiento local } \\
\text { Atracción de proveedores de material directo } \\
\text { Proyectos conjuntos con universidades para } \\
\text { formación profesional }\end{array}$ & $\begin{array}{l}\text { Autonomía en la toma } \\
\text { de decisiones respecto a } \\
\text { productos, abastecimiento } \\
\text { de material directo } \\
\text { e indirecto, nuevos } \\
\text { productos }\end{array}$ \\
\hline - Establecimiento de grupos de trabajo & $\begin{array}{l}\text { Delegación en la toma de algunas decisiones } \\
\text { sobre diseños, clientes, proveedores e } \\
\text { instituciones }\end{array}$ & $\begin{array}{l}\text { Autonomía en la toma de } \\
\text { decisiones } \\
\text { respecto a productos, } \\
\text { abastecimiento de } \\
\text { material directo e indirecto, } \\
\text { nuevos productos }\end{array}$ \\
\hline $\begin{array}{l}\text { Copia y adaptaciones menores de } \\
\text { especificaciones de equipos de prueba } \\
\text { existentes } \\
\text { Reconstrucción de equipos pequeños sin } \\
\text { asistencia técnica } \\
\text { - Mantenimiento básico programado }\end{array}$ & $\begin{array}{l}\text { Adaptaciones a grandes equipos } \\
\text { Ingeniería en reversa } \\
\text { Ingeniería y construcción de equipo de prueba } \\
\text { Mantenimiento preventivo }\end{array}$ & $\begin{array}{l}\text { Diseño y construcción de } \\
\text { equipos y componentes } \\
\text { Investigación y desarrollo } \\
\text { para nuevos componentes }\end{array}$ \\
\hline
\end{tabular}

Fuente: Elaboración propia. 
Así fue posible identificar y determinar el nivel de las actividades, mejoras y resultados obtenidos. Del reconocimiento del potencial de contribución de la información operativa cotidiana al proceso de asimilación de la tecnología, se desprende la formulación de las cuatro capacidades para el fortalecimiento para un programa de aprendizaje tecnológico ${ }^{21}$ y construcción de capacidades.

De acuerdo con las evidencias obtenidas, el grupo industrial ha acumulado capacidades tecnológicas de diferentes niveles. Desde las operativas básicas hasta las innovativas avanzadas, en las funciones técnicas de inversión, producción y soporte, como se muestra en la matriz de capacidades tecnológicas del grupo. Es decir, que ha conseguido aprender de manera importante para su progreso y subsistencia en un mercado.

\section{Construcción de capacidades tecnológicas: el análisis de las funciones técnicas}

De las dos funciones técnicas señaladas por Bell y Pavitt esta investigación recogió amplia evidencia sobre las actividades relacionadas con las variables toma de decisiones y control, y la preparación y ejecución de proyectos.

Las capacidades tecnológicas identificadas en la empresa, correspondientes a esta función técnica, se examinaron a partir de diversas actividades. ${ }^{22}$ En su conjunto, se observa que el Grupo San ha alcanzado un nivel de capacidades innovativas básicas

${ }^{21}$ Entendido el aprendizaje tecnológico como un proceso dinámico que parte de los niveles de conocimiento alcanzados mediante la adquisición de elementos de información tecnológica, durante el proceso de negociación y contratación y que, una vez iniciado el proceso productivo, adquiere vitalidad sólo a través del contraste permanente entre las directrices operativas adquiridas y las expectativas de comportamiento teórico del proceso, por un lado, y, por otro, de los resultados evidenciados mediante la observación sistemática de la respuesta real de las instalaciones.

22 Abarcan estudios de factibilidad, programación de actividades, adaptaciones en el equipo y maquinaria, desarrollo de nuevas prácticas de operación, diseño y desarrollo de nuevos componentes, selección de tecnología y proveedores, adquisición e instalación de la bóveda del horno de afino, reconstrucción de equipos pequeños sin asistencia técnica, diseño y construcción de equipo, investigación para nuevos componentes, desarrollo de nuevos sistemas de producción, diseño incremental del producto, desarrollo de prácticas de aceros especiales, capacitación sobre el uso de aceros limpios, búsqueda, evaluación y selección de tecnología, búsqueda, evaluación y selección de proveedores, negociación con proveedores. Administración de proyectos completos, desarrollo de nuevos sistemas de producción y componentes, adquisición de equipo, estudios del medio ambiente, administración y seguimiento de los proyectos, designación de grupos de trabajo, reclutamiento, capacitación, puesta en marcha de nueva tecnología en el proceso de producción. 
e intermedias ${ }^{23}$ en las variables toma de decisión y control, preparación y ejecución del proyecto; y de manera significativa, se encontraron innovaciones avanzadas en estas variables por el número de actividades reportadas, relacionadas con diseño de procesos y desarrollo de investigación para nuevos productos, principalmente.

\subsection{Funciones técnicas de producción}

En las funciones técnicas de producción el Grupo San ha logrado desarrollar capacidades innovativas avanzadas, tanto en el proceso como en el producto.

Referente a las capacidades centradas en el proceso, éstas se identificaron a partir de actividades señaladas en las tablas de resultados por área como: mejora del layout, ${ }^{24}$ programa de mantenimiento productivo total, ${ }^{25}$ mejoras en el funcionamiento de la maquinaria ${ }^{26}$ y mejoras en aprovechamiento de la materia prima al optimizar el uso y suministro de materiales directos e indirectos, adaptaciones menores al proceso basado en tiempos y movimientos, implementación de técnicas de alotamiento ${ }^{27}$ y optimización de materiales, aumento de la capacidad de producción (basado en el

\footnotetext{
${ }^{23}$ De acuerdo con Bell y Pavitt (1995), la habilidad para negociar con proveedores, habiendo realizado procesos de búsqueda, evaluación y selección, se asocia con una capacidad innovativa intermedia. En el caso del Grupo San, no sólo monitorea a sus proveedores, sino que además da seguimiento y retroalimentación con el fin de conseguir un mejor beneficio para la empresa, a través del proceso de negociación. Con base en lo anterior, se puede decir que esta capacidad se ha fortalecido a través de los mecanismos de aprendizaje: interacción con proveedores, vigía tecnológica y el sistema de calidad y mejora continua.

${ }^{24}$ Esta actividad consistió principalmente en hacer una distribución de planta, lo que permitió reducir los desplazamientos del personal y de los materiales, mejorar los flujos de la materia prima, disminuir el tiempo total de proceso, disminuir los retrocesos, mejorar la ubicación de las máquinas y, en general, mejorar la logística de producción de la empresa.

${ }^{25}$ Este programa de mantenimiento productivo total consistió en planear la revisión a las diferentes máquinas que tiene la empresa, con el fin de evitar interrupciones en el proceso productivo, por daños o desgaste. Uno de los mecanismos de aprendizaje tecnológico que más ha aportado a esta capacidad, logrando una mayor evolución de la misma, ha sido el sistema de calidad y mejora continua. Este sistema de calidad ha obligado a la empresa a pasar de hacer un mantenimiento, en muchas ocasiones correctivo, a un mantenimiento programado, que le garantiza al Grupo San una mayor seguridad en su proceso productivo.

${ }^{26}$ Esta actividad consistió en modificar la maquinaria o el equipo con el fin de lograr un mejor funcionamiento de éstos. El personal del Grupo San ha desarrollado una gran habilidad para realizar adaptaciones a la maquinaria que tiene la empresa, con el fin de adecuarla a las necesidades y en algunas ocasiones para mejorar el proceso. Esta habilidad para mejorar las especificaciones de las máquinas es considerada en este análisis como una capacidad innovativa intermedia, ya que la empresa ha pasado de hacer adaptaciones menores a la maquinaria a hacer mejoras por encima de las especificaciones del proveedor. En este sentido, el grupo ha desarrollado un mecanismo de aprendizaje muy importante, como es el de mejoras a maquinaria y equipo.

${ }^{27}$ Formar lotes de chatarra con determinadas características químicas.
} 
balanceo de líneas) mejoras al proceso al crecer el número de recargues, mejoras en el rendimiento de materiales directos e indirectos, rediseño y diseño de partes del proceso, ensamble y manufactura, validación de procesos, estiramiento de la capacidad de producción, sistemas de calidad y mejora continua, innovaciones y desarrollo de nuevos procesos.

Referente a las capacidades centradas en el producto, ${ }^{28}$ el Grupo San ha logrado desarrollar capacidades con características que le permiten fabricar sus productos, rebasando los requerimientos planteados por sus clientes. Por lo tanto, los mecanismos de aprendizaje tales como: el sistema de calidad y mejora continua, interacción con proveedores y vigía tecnológica, entre otros, sobresalen como elementos importantes en la construcción de capacidades tecnológicas en la empresa.

\subsection{Funciones técnicas de soporte}

Las funciones técnicas de soporte abarcan la vinculación externa e interna, así como el desarrollo de equipo.

3.2.1 La vinculación externa tuvo lugar al establecer relaciones con clientes a través de las especificaciones del producto, búsqueda y negociación con proveedores de materiales directos e indirectos, búsqueda de vínculos con instituciones de educación media superior y superior, para entrenamiento de personal, transferencia de tecnología a proveedores para incrementar eficiencia, calidad y abastecimiento, atracción de proveedores de materias primas y materiales indirectos, proyectos conjuntos con instituciones para formación profesional, autonomía en la toma de decisiones respecto al abastecimiento de suministros. Las capacidades de vinculación externa se han construido mediante los mecanismos de aprendizaje con proveedores, visitas a ferias internacionales, vigía tecnológica y vínculos con universidades y centros de investigación. Estos mecanismos le han servido a la empresa para adquirir información y conocimiento nuevo sobre proveedores, clientes e instituciones locales, regionales e internacionales. Por lo tanto, con las capacidades que se generan a partir de ellos, la empresa ya no sólo usa y opera la tecnología existente, sino que es capaz de generar y administrar cambio técnico de nivel básico.

3.2.2 La vinculación interna abarca el establecimiento de grupos de trabajo, delegar en la toma de decisiones sobre proveedores, capacitación interna a operarios y super-

${ }^{28}$ Abarca las actividades de obtención de la certificación del proceso, adaptaciones menores al producto de acuerdo a necesidades del cliente, el mejoramiento en las especificaciones del producto, mejoras incrementales en la calidad del producto, diseño incremental de los productos, diseño de características básicas de nuevos productos e innovaciones de productos, desarrollo de fórmulas nuevas de carbón para satisfacer necesidades del mercado. 
visores de las áreas productivas y el desarrollo de nuevas prácticas de operación. En particular el desarrollo de equipo incluye copia y adaptaciones menores de especificaciones de los equipos de prueba existentes, reconstrucción de equipos pequeños sin asistencia técnica, mantenimiento básico programado, diseño y construcción de equipo, adaptaciones a grandes equipos, ingeniería en reversa, ingeniería y construcción de equipo de prueba, mantenimiento preventivo, diseño y construcción de equipos y componentes, investigación y desarrollo para nuevos componentes, rediseño de partes del proceso y manufactura.

\section{Actividades y mecanismos de aprendizaje y acumulación de capacidades internas}

Las actividades y mecanismos de aprendizaje son internos (capacitación, mejora y rutina) y externos (proveedores, competidores y universidades y centros de investigación).

\subsection{Internas: capacitación, mejora continua y rutinas en la producción}

Los mecanismos de aprendizaje tecnológico se definen como aquellas actividades que se desarrollan en la empresa y que les permiten a los individuos y grupos de individuos asimilar conocimiento nuevo y desarrollar habilidades para contribuir a la construcción de capacidades tecnológicas. Las actividades y los mecanismos y las formas de aprendizaje tecnológico que han permitido alcanzar el escalamiento productivo y tecnológico logrado por la empresa se detallan a continuación. ${ }^{29}$

La capacitación permitió a la empresa acumular conocimiento. Lo importante de los programas de capacitación implementados radica en la motivación que se les da a los operarios para que se interesen en los procesos productivos, para que se integren y se sientan arraigados, además de los estímulos económicos por los resultados logrados en la producción. El resultado de ello fue un mejor desempeño de operarios y técnicos al momento de exponer y proponer soluciones ante los problemas eventuales en los distintos puntos de las líneas de producción e incluso en proponer mejoras en materiales, procesos y producto.

El programa de inducción es aplicado al personal que ingresa por primera vez a la empresa y está integrado por tres bloques de información de carácter introductorio:

\footnotetext{
${ }^{29}$ Es importante aclarar que el Grupo San no ha logrado un desarrollo homogéneo de muchos de estos mecanismos en sus plantas. Lo anterior se debe a que las plantas tienen diferentes niveles de complejidad, lo que dificulta la implantación de dichos mecanismos. 
inducción genérica, entrenamiento básico y entrenamiento de especialidad. Este programa le permite a la empresa mejorar el uso de las habilidades de sus trabajadores y disminuye la incertidumbre de quienes se vinculan por primera vez con ellas logrando así una mejor eficiencia en el desempeño del trabajo.

Los programas de capacitación que ha desarrollado el Grupo San se resumen básicamente en cuatro: Programa de educación básica, Formación multifuncional, Programa de innovación tecnológica y Cursos especiales. Estos programas se vuelven mecanismos de aprendizaje para la empresa, ya que a través de ellos las personas obtienen nuevo conocimiento que les permite realizar mejor su trabajo, aumentar su iniciativa para lograr buenas destrezas en el trabajo y los prepara para ocupar otros puestos.

Los procesos de control de calidad y mejora continua permitieron desarrollar habilidades sistemáticas para responder adecuadamente a los problemas que se presenten. La calidad es una actividad de soporte que se practicó en cada una de las áreas productivas, con la finalidad de responsabilizar al personal de las mejoras en el proceso y de la calidad del producto final. Al respecto la empresa tiene establecida la práctica de revisar, actualizar y documentar los nuevos procedimientos implementados, los cuales incluyen las mejoras realizadas. Asimismo, se actualiza la nomenclatura tanto de documentos como de registros de manera que está en condiciones de ser auditada por aseguramiento de calidad.

El sistema de calidad es un proceso mediante el cual la empresa asegura que sus productos cumplen con las especificaciones que exige el cliente. Con base en el tipo de producto que la empresa fabrica, y en el tipo de cliente que atiende, el sistema de calidad que ha desarrollado la empresa se convirtió en uno de los mecanismos de aprendizaje más importante, porque le permite aprender, pues a través de este sistema las personas que trabajan asimilan conocimiento y pueden desarrollar nuevas habilidades que redundan en un mejor desempeño.

En lo que se refiere al aprendizaje por experiencia en la producción, la empresa ha respondido rápido a las exigencias del mercado, mediante el aprendizaje en la planeación de la producción. En ese sentido, se requieren habilidades como la capacidad de los operarios para intensificar las actividades de producción, mantenimiento y ajuste, y de los técnicos para supervisar una mayor rotación en el uso de herramientas, equipos y maquinaria.

Los cambios que se llevaron a cabo no podrían haber sido posibles sin una comprensión profunda del proceso de producción y de las posibilidades y limitaciones del equipo en operación. Esta comprensión se adquirió básicamente de las experiencias 
de producción al nivel de planta. Así, el proceso de aumentar la capacidad fue resuelto por personal de la planta (técnicos y operarios). El proceso fue de ensayo y error y de aprendizaje de la experiencia.

Las mejoras a maquinaria y equipo se plasman en el "aprender haciendo" y "aprender usando”. Por este medio la empresa ha logrado acumular lo que actualmente el personal domina, es decir, todo un conjunto de experiencias y habilidades desarrolladas a través del tiempo. Estos procesos de aprendizaje tecnológico fueron impulsados principalmente por la necesidad de alcanzar objetivos de producción y eficiencia, lo que permitió lograr perfeccionamientos en los equipos y máquinas de gran impacto en la productividad, como se puede evidenciar en los resultados alcanzados en los parámetros operacionales. La motivación central no fue la de desarrollar tecnología per se, sino alcanzar los objetivos y metas de producción y calidad exigidos por el mercado. Sin embargo, este esfuerzo dio como resultado el desarrollo de capacidades tecnológicas nuevas y especificas de la empresa.

\subsection{Externas: proveedores, competidores y universidades y centros de investigación}

El aprendizaje por interacción con proveedores fue una de las actividades de aprendizaje más importante para la innovación. Uno de los resultados de esta interacción fue el aprendizaje del personal en el diseño y mejora de materiales indirectos, funcionamiento y mantenimiento de equipos y maquinaria sin necesidad de acudir a ingenieros o técnicos extranjeros.

La relación con proveedores es muy importante porque, de acuerdo a las evidencias encontradas en este estudio, muchas innovaciones realizadas surgieron a partir de la relación con los proveedores, en especial los de refractarios, de ferroaleaciones, microaleados, accesorios de maquinaria y equipo. Como resultado de esta interacción con los proveedores (aprender interactuando), el Grupo San ha conseguido optimizar de manera significativa los rendimientos de los materiales indirectos (como es el caso concreto de los refractarios), e insumos utilizados en sus procesos y ha perfeccionado considerablemente la calidad de sus productos, logrando producir aceros más limpios y aceros especiales. Lo anterior, también se refleja en notables mejoras al proceso, incrementando la productividad reflejada en los resultados registrados en los parámetros operacionales.

La visita a los establecimientos de los competidores y por rotación de personal es un tipo de aprendizaje más informal. Los ingenieros y técnicos locales visitan empresas en diferentes puntos de manera informal para ver cómo realizan un determinado pro- 
ceso. Mediante estas visitas se aprende de las experiencias de los competidores sin recurrir a algún programa de capacitación o entrenamiento formal.

La vigilancia tecnológica consiste en enviar personas de considerable experiencia y conocimiento del proceso productivo a diferentes partes del mundo, para que investiguen todo lo relacionado con las innovaciones que hay en el sector siderúrgico y que sean susceptibles de aplicar. Con esta práctica se ha conseguido capturar información de mucho valor para la empresa. Es por ello que se considera como un mecanismo de aprendizaje tecnológico.

La vinculación con instituciones de educación media superior y superior ha sido importante no sólo como medio de aprendizaje del personal de las plantas y de los estudiantes de las instituciones de educación técnica media y superior, sino también como medio de transferencia de tecnología de la empresa hacia su entorno.

Las actividades por prácticas y pruebas, sobre todo con refractarios con el fin de mejorar el rendimiento del uso de ferroligas y fundentes; prácticas operativas para nuevas calidades de acero desarrolladas por la empresa en estudio; pruebas con el turbostop en la máquina de colada continua, para fabricación de aceros de alta limpieza, muestran una evolución muy significativa, en cuanto al desarrollo de nuevas destrezas para mejorar los procesos y productos. Se identifica claramente en estas actividades la forma de aprendizaje tecnológico propuesta por Stiglitz (1987), en la que sugiere que el aprendizaje constituye una habilidad especializada que se desarrolla dentro del propio proceso de trabajo que la denomina "aprender a aprender- learning to learn", es decir, desarrollando la habilidad de apropiarse de hábitos nuevos, sustituyendo formas de saber hacer menos eficientes.

La vinculación con instituciones de educación superior, media superior y centros de investigación ha sido un mecanismo de aprendizaje aprovechado para la capacitación de personal operativo, así como para la investigación orientada especialmente a perfeccionar los análisis metalográficos, mejora de implementos de maquinaria y pruebas de laboratorio en las funciones técnicas de producción y soporte. El aprender de manera colectiva, asimilando y compartiendo el conocimiento entre el personal y las IES y centros de investigación, ha incidido favorablemente en mejoras de las habilidades de sus trabajadores.

\section{Conclusiones}

En este artículo se analizaron los procesos de aprendizaje tecnológico a nivel local en una empresa siderúrgica en México, con base en una metodología que utiliza ins- 
trumentos de análisis basados en actividades y las funciones técnicas de inversión, producción o de soporte de una empresa. Con el análisis efectuado se desprenden las siguientes conclusiones.

El Grupo San ha acumulado capacidades tecnológicas que evidencian un mayor aprendizaje y niveles de complejidad, esto es, puede decirse que ha avanzado de operaciones básicas hasta las innovativas avanzadas. El aprendizaje tecnológico se logra al desarrollar cambios técnicos en su mayoría de manera cotidiana, como son los procesos de compras, adaptación y mejora de equipos y procesos, localizar y solucionar fallas, expandir las plantas o desarrollar procesos o productos nuevos. El aprendizaje no es necesariamente el objetivo perseguido al desarrollar estas actividades, sin embargo, se pueden aprovechar estas actividades de cambio técnico, no sólo para resolver los problemas de producción sino también para generar un resultado de aprendizaje.

El aprendizaje tecnológico se relaciona estrechamente con la introducción de nuevos productos propiciando la innovación y el cambio tecnológico, dando lugar a nuevas tecnologías, procesos, sistemas productivos, organización del trabajo y soluciones técnicas. Es el aprendizaje de un conocimiento codificado en manuales, diagramas, planos, software, instrumentos de producción, sistemas de manufactura y de calidad, procesos y técnicas, interacciones formales e informales con los propios trabajadores, proveedores y clientes, facilitado por el flujo de información técnica como resultado de la interacción y por las estrategias de capacitación en y fuera de la empresa.

El aprendizaje se presenta en un ambiente específico de interacciones humanas de una organización a otra y de un individuo a otro, donde se asimila una cultura tecnológica que se concreta en las características de la distribución del trabajo y, más extensamente, en las características tecnológicas de la empresa, dando lugar a aprendizajes particulares, específicos y aun exclusivos con un carácter acumulativo.

La estrecha interacción entre las actividades de producción da una modalidad al aprendizaje tecnológico, caracterizado por una variedad de conocimientos interdisciplinarios, habilidades y actitudes que fortalecen el desarrollo competitivo. Los aprendizajes más relevantes en la dimensión técnica (funciones técnicas) se presentan en la adaptación de tecnologías, modificación de equipos, diseño y rediseño de productos. En cambio, el aprendizaje tecnológico, al instalar una planta nueva, se relaciona con la asimilación y adaptación de nuevas tecnologías, con el conocimiento general de la planta, los sistemas de calidad, las tecnologías de información y las actitudes derivadas de la experiencia, el trabajo en equipo y la orientación al cliente. La relación proveedor-cliente no sólo se aplica entre empresas, sino entre áreas de la misma 
empresa y entre personal de las propias áreas, esto es, todos los participantes son usuarios y proveedores.

El proceso de aprendizaje tecnológico incide de manera directa en la construcción gradual de capacidades tecnológicas en el Grupo San. Esto se observa en la importante relación que existe entre las actividades, mecanismos y formas de aprendizaje y las capacidades tecnológicas construidas en las distintas áreas del proceso productivo.

El Grupo San ha logrado aprender de manera importante para su desarrollo y supervivencia en un mercado que se caracteriza por condiciones bastante exigentes; asimismo, se puede precisar que la empresa ha logrado desarrollar prácticas exitosas que contribuyen a la construcción de capacidades tecnológicas y que se han transformado en mecanismos de aprendizaje. Por otro lado, a pesar de haber logrado un nivel importante de acumulación de conocimiento, aún no alcanza un nivel óptimo que le permita mejorar su competitividad. Por lo tanto, es de vital importancia que la empresa se comprometa más activa y estratégicamente para mejorar su proceso de aprendizaje tecnológico, mejorando muchas de sus prácticas tradicionales y la cultura organizacional que actualmente predomina.

\section{Bibliografía}

Arrow, K. ( 1962), The Economic Implications of Learning by Doing. Review of Economic Studies (pp. 155-173) vol. 29.

Bell, M. y K. Pavitt (1993), Technological Accumulation and Industrial Growth: Contrasts Between Developed and Developing Countries. En Science Policy Research (pp. 157-209).

(1995), The Development of Technological Capabilities. En I. U. Haque (ed.), Trade, Technology and International Competitiveness (pp. 69101). Washington: The World Bank.

Bell, Martin y Keith Pavitt (1992), Accumulating technological capability in developing countries. Washington: The World Bank Research Observer.

Cyert, R.M. y J. G. March (1963), A Behavioral Theory of the Firm, Nueva Jersey: Prentice Hall.

Dosi, G. et al. (1988), Technical Change and Economic Theory, Pinter Publisher.

Dosi, Giovanni, R. Nelson, y S. Winter (2000), The Nature and Dynamics of Organizational Capabilities.New York: Oxford Press. 
Dutrenit, G. y M. CAPDEVIELle (1993), El perfil tecnológico de la industria mexicana y su dinámica innovadora en la década de los ochenta. El trimestre económico, vol. 60(3), núm. 239, julio-septiembre.

JAsso, J. (2004), Tecnología y organizaciones: consideraciones acerca de una propuesta teórica de la innovación. En Revista del Colegio de San Luis, año VI, núm. 18, septiembre-diciembre.

KaTz, J. (1989), La teoría del cambio tecnológico y su adecuación al caso de los países de industrialización tardía. En CEPAL, Estudios e Informes de la CEPAL, LC/G.1493, noviembre.

KIM, L. (1997), From Imitation to Innovation. The Dynamics of Korea's Technological Learning, Boston: Harvard Business School Press.

LALL, S. (1987), Learning to industrialize: the acquisition of technological capability by India. London: MacMillan Press.

Lall, Sanjaya (1992), Technological Capabilities and Industrialization. En World Development (pp. 165-186), vol. 20, núm. 2.

(1996), Las Capacidades Tecnológicas. En Salomón, Jean-Jacques, F. Sagasti y C. Sachs (c omps.), Una Búsqueda Incierta, Ciencia, Tecnología y Desarrollo, El Trimestre Económico, (pp. 301-342) lectura núm. 82.

Malecky, Eduard J. (1997), Technology and Economic Development. The Dynamics of local, Regional and National Competitiveness, Longman.

PavitT, K. (1984), Patterns of technical change: towards a taxonomy and a theory, Research Policy, vol. 13, núm. 6.

Romis, H. (1999), Acquisition of Technological Capability in Small Firms in Developing Countries. Londres: MacMillan.

Rosenberg, N. (comp.) (1979), Economía del Cambio Tecnológico. México: FCE.

STiglitz, J. (1987), Learning to learn, localised learning and technological progress. En P. Dasgupta y P. Stoneman (ed.), Economic Policy and Technology Performance. Cambridge, Cambridge: University Press.

Tellis, Winston (1997a), Introduction to case study. En The Qualitative Report, 3. Consultado el 31 de julio de 2003 desde http://www.nova.edu/ssss/QR/QR33/tellis1.html 
Tellis, Winston (1997b), Application of a case study methodology. En The Qualitative Report,3.Consultado el 31 de julio de 2003 en: http://www.nova.edu/ ssss/QR/QR3-3/tellis2.html

Tremblay, P. (1998), Technological Capability and Productivity Growth: An Industrialized/Industrializing Country Comparison, Montreal, Centre Interuniversitaire de Recherche en Analyse des Organisations.

WestPhal, L., K. Kritayakirana et al. (1990), The development of technological capability in manufacturing: a macroscopic approach to policy research. En R. Evenson y G. Ranis, Science and Technology: Lessons for Development Policy, Londres: Intermediate Technology Publications.

Wignaraja, G. (1998), Trade Liberalisation in Sri Lanka: Exports,Technology and Industrial Policy. Londres: MacMillan.

(2001), Firm Size, Technological Capabilities and Market Oriented Policies in Mauritius, Discussion papers, núm. 1. Maastricht: Universidad de las Naciones Unidas (UNU)/Instituto de Nuevas Tecnologías.

YIN, R. K. (1994), Case Study Research. Design and Methods (2a. ed.). California: Applied Social Research Methods Series, Sage Publications.

G 